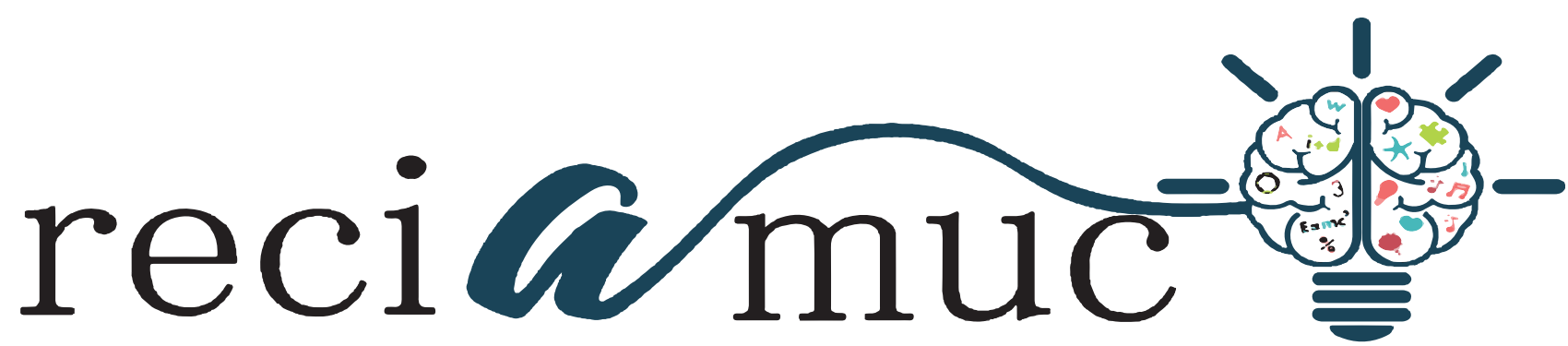

DOI: 10.26820/reciamuc/4.(2).abril.2020.241-250

URL: https://reciamuc.com/index.php/RECIAMUC/article/view/519

EDITORIAL: Saberes del Conocimiento

REVISTA: RECIAMUC

ISSN: 2588-0748

TIPO DE INVESTIGACIÓN: Artículo de Revisión

CÓDIGO UNESCO: 3213 Cirugía

PAGINAS: $241-250$

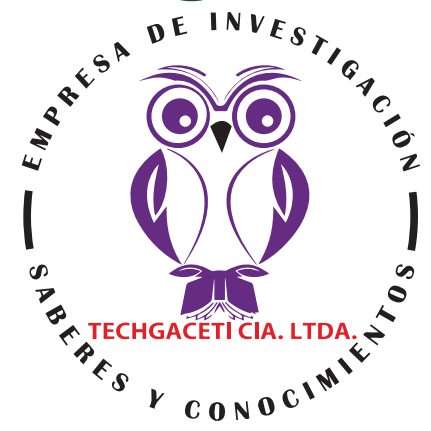

\title{
Una evaluación de la curva de aprendizaje en cirugía general robótica
}

An evaluation of the learning curve in robotic general surgery

Uma avaliação da curva de aprendizado em cirurgia geral robótica Jorge Enrique García Delgado; Gabriela Stefanía Morales Chaucaláa; Hugo Jofre Vera Molina ${ }^{3}$

RECIBIDO: 18/01/2020 ACEPTADO: 20/03/2020 PUBLICADO: 30/04/2020

1. Médico; Semedic Centro. Director Tecnico; Guayaquil, Ecuador; jorgequique00@hotmail.com; (D) https://orcid. org/0000-0001-8565-6671

2. Médico; Investigador Independiente; Guayaquil, Ecuador; dragabrielamorales@hotmail.com; (D) https://orcid. org/0000-0001-6606-5219

3. Doctor en Medicina y Cirugía Universidad Estatal de Guayaquil; Especialista en Ginecobstetricia Universidad Estatal de Guayaquil; Especialista en Medicina del Trabajo Colegio de Médicos del Guayas; Diplomado Superior en Salud Escolar y de Adolescentes Universidad Estatal de Guayaquil; Coordinador Médico Semedic; Presidente de la Sociedad Ecuatoriana de Medicina Escolar y de Adolescentes; Guayaquil, Ecuador; papihugo64@outlook.com; D https://orcid.org/0000-0003-4568-5384

CORRESPONDENCIA

Jorge Enrique García Delgado

jorgequique00@hotmail.com

Guayaquil, Ecuador

(c) RECIAMUC; Editorial Saberes del Conocimiento, 2020 


\section{RESUMEN}

La Cirugía Robótica se ha presentado en la Medicina como el tratamiento más acertado para ciertas patologías, logrando exitosos resultados. La demanda del aprendizaje de esta nueva tecnología cada día es mayor, por lo que se han elaborado estrategias de enseñanzas que aceleren el aprendizaje. Los estudios analizados en este artículo han demostrado que la Curva de Aprendizaje en la Cirugía General robótica, es factiblemente aplanada en el tiempo a medida que se realizan los casos quirúrgicos.

Palabras clave: Cirugía, Robótica, Curva de Aprendizaje, Da Vinci.

\section{ABSTRACT}

Robotic Surgery has been presented in Medicine as the most successful treatment for certain pathologies, achieving successful results. The demand for learning this new technology is increasing every day, which is why teaching strategies have been developed to accelerate learning. The studies analyzed in this article have shown that the Learning Curve in robotic General Surgery is feasibly flattened over time as surgical cases are performed.

Keywords: Surgery, Robotics, Learning Curve, Da Vinci.

\section{RESUMO}

A Cirurgia Robótica tem se apresentado na Medicina como o tratamento de maior sucesso para determinadas patologias, obtendo resultados exitosos. A demanda pelo aprendizado dessa nova tecnologia aumenta a cada dia, por isso estratégias de ensino têm sido desenvolvidas para acelerar o aprendizado. Os estudos analisados neste artigo mostraram que a Curva de Aprendizado em Cirurgia Geral Robótica é viável achatada com o tempo conforme os casos cirúrgicos são realizados.

Palavras-chave: Cirurgia, Robótica, Curva de Aprendizagem, Da Vinci. 


\section{Introducción}

La robótica es una tendencia indetenible en todas las áreas y dimensiones humanas, por lo que la medicina, específicamente la quirúrgica, no está excluida de la influencia de ésta y de ninguna nueva tecnología.

Es un hecho fáctico que la cirugía robótica es la opción de tratamiento más adecuado por presentar resultados satisfactorios a los pacientes. Minimiza el riesgo de intervención y garantiza una óptima recuperación en el menor tiempo posible. Abarca e impacta las especialidades médicas, porque no sólo su intervención es mínima, sino que alcanza lugares pequeños a los que no podría llegar el brazo o la mano humana. (Minor, Mosso, Domínguez, \& et al, 2002)

No obstante, requiere de una especialidad y capacitación bastante elevada, por lo cual, una revolución de paradigmas en las enseñanzas médicas (en éste caso, en el área quirúrgica), es inevitable. Aunque en la actualidad ya se cuenta con complejos sistemas y programas diseñados para enseñar virtualmente sin poner en riesgo la vida del paciente, aun no es suficiente la manera más correcta de capacitar y enseñar en el menor tiempo posible (Herrel SD, 2005).

En efecto, las habilidades de un cirujano no sólo consisten en aprender manejar excelentemente una maquinaría de alta tecnología, pues el conocimiento, la experticia y la humanidad dependen del profesional per se, de tal forma que estas herramientas tecnológicas deben ser correctamente manejadas con las cualidades mencionadas, de allí la importancia de su adecuado proceso aprendizaje.

Cuando se habla de curva de aprendizaje en medicina, se relaciona con la experiencia y el tiempo. Dos variables que parecen definitorias por sí solas, pero que en definitiva, siguen dependiendo de la capacidad del profesional, ya que un cirujano puede haber operado muchísimas veces y ganar experiencia en un corto tiempo, así mismo aquel que en un largo tiempo haya realizado la misma cantidad de operaciones, y pudo haber ganado la misma experiencia (Ahlering , 2003).

Por otro lado, realizar un tipo de intervención quirúrgica muchas veces, no garantiza que se halla hecho excelentemente, o aceptable. Por lo que, sin duda alguna, la cualidad del cirujano juega un factor importante en el proceso de aprendizaje. Y aunque el robot es una ventaja potencial para disminuir errores humanos, es necesario aprender cómo funciona y la mejor forma de manejarlo.

El presente artículo presenta evaluaciones importantes sobre la curva de aprendizaje en cirugía general robótica de los estudios más relevantes que utilizaron el Da Vinci ${ }^{\circledR}$ Surgical System. La selección primaría fue con este tipo de robot por ser el más utilizado en la actualidad, con el que se han realizado más cirugías alrededor del mundo.

De esta manera se podrá mostrar un análisis informativo sobre la actual realidad de la curva de aprendizaje de la cirugía general robótica, no sin antes esbozar la historia y actualidad de la inserción de los robots en la medicina, y la aproximación hacia la definición más aceptable para medir la curva de aprendizaje.

\section{Método}

El presente artículo describe y compara diferentes evaluaciones de curvas de aprendizaje, en diferentes cirugías generales. Se recolecta esta información de diferentes fuentes bibliográficas de estudios relacionados a la robótica como apoyo quirúrgico, y su curva de aprendizaje asociada. Dichas fuentes bien provienen de diferentes revistas digitales como de fuentes directas.

Tomando en cuenta que en la actualidad, el robot Da Vinci® Surgical System es el más utilizado a nivel mundial, se ha seleccionado solamente los estudios que le usaron en 
sus procedimientos quirúrgicos, ya que la data sobre la curva de aprendizaje es idónea para el propósito de evaluar la curva de aprendizaje de algunos cirujanos de diferentes lugares del mundo que utilizan este robot. Para un posterior análisis de las observaciones y comparaciones.

\section{Resultados}

\section{La Robótica en la Medicina}

(1) La robótica inicia su integración a la biolof gía en 1980 con los estudios de Curl, Kroto y

Smalley. Luego comenzó a usarse en medicina principalmente después del surgimiento de la cirugía mínimamente invasiva. Durante los primeros años de esta década, se realizaron experimentos quirúrgicos con robots en neurocirugía y ortopedia, siendo en 1985 cuando el robot PUMA 560 logró introducir una aguja en el cerebro mediante una guía por Tomografía Axial Computarizada (TAC) (Pereira Fraga, 2017).

Para 1995 la Computer Motion INC, terminó su prototipo de robot cirujano Zeus y fue probado y mejorado hasta llegar a ser el AESOP 4000, hasta la actualidad disponible como un abrazo robotizado inteligente controlado por reconocimiento de voz previa configuración del cirujano (Galeano, 2014).

En 1997 el Intuitive Surgical Inc Termina el prototipo de nombre MONA, con brazos independientes para que un cirujano remoto pudiese controlarlos, utilizando un sistema amo-esclavo con consola de mandos. Fue el antecesor de Da Vinci Surgical System, que surgió en 1999 y fue el primero en obtener la aprobación de la FDA (Food and Drug Administration, por sus siglas en inglés) en el año 2000, para realizar cirugías abdominales por vía laparoscopia. A partir de éste punto histórico se multiplicaron las creaciones robóticas de apoyo cirujano que mejoraron el tamaño y las delicadas conexiones dentro de quirófano que requería Da Vinci, tales como robots asistentes-coordinadores
(Hermes), servo-asistentes (AESOP y otros brazos asistentes), efectores semiautónomos (Robodoc, Caspar, Ac-robot, Probot, PAKI) y los robots tele controlados como el Da Vinci (Morris B, 2005).

\section{Da Vinci Surgical System}

La aplicación de la robótica en las diferentes técnicas quirúrgicas consiste en el tratamiento de un paciente mediante robots especializados manejados por un cirujano, y dependiendo del tipo de robot, la cirugía puede ser (Ballantyne G, 2002):

- Robótica: Cuando los robots son directamente controlados por el cirujano, quien se encuentra cerca de la mesa de cirugía.

- Telerrobótica: Cuando el cirujano opera en un sitio diferente de donde se encuentra el paciente utilizando plataforma quirúrgica con instrumentos de robóticos.

- Telepresencia: Cuando el cirujano utiliza un telerrobot para transmitir los movimientos de las manos del cirujano al quirófano remoto sin necesidad de ver al paciente, proyectando virtualmente las imágenes del campo operatorio a un sitio remoto.

- Teleasesoramiento: Se aplica este término cuando un cirujano experto localizado en hospital, enseña a otro cirujano en un sitio diferente. Se utiliza para crear aulas virtuales.

Da Vinci, el primer proyecto de telepresencia a corta distancia, es un sistema maestro- esclavo de telepresencia. Del lado del paciente, en la mesa de operaciones, se encuentra el carro que sostiene los instrumentos y el brazo de la cámara. Con un sistema denominado centro de control, que hace que se ejerza una mínima presión sobre la pared del cuerpo del paciente. Por otro lado, consta de una consola con un endoscopio 3D y mandos EndoWrist, diseñados para que los cirujanos mantengan sus 
destrezas naturales, proporcionándole mayor rango de movimiento que el que posee la mano humana, con pulso exacto. Sirven para trabajar de forma más rápidas y precisas con las suturas, disecciones y técnicas de manipulación de tejidos que existen. Los dedos de las manos se ajustan a los controles que asemejan a un par de guantes. Los instrumentos intracorpóreos se mueven tal cual se mueven las manos del cirujano. Los pies controlan pedales que controlan funciones de los botones manuales, mientras el cirujano ve el campo quirúrgico y sus manos, como instrumentos, tal como se muestra en la imagen 1 (Minor, Mosso, Domínguez, \& et al, 2002).

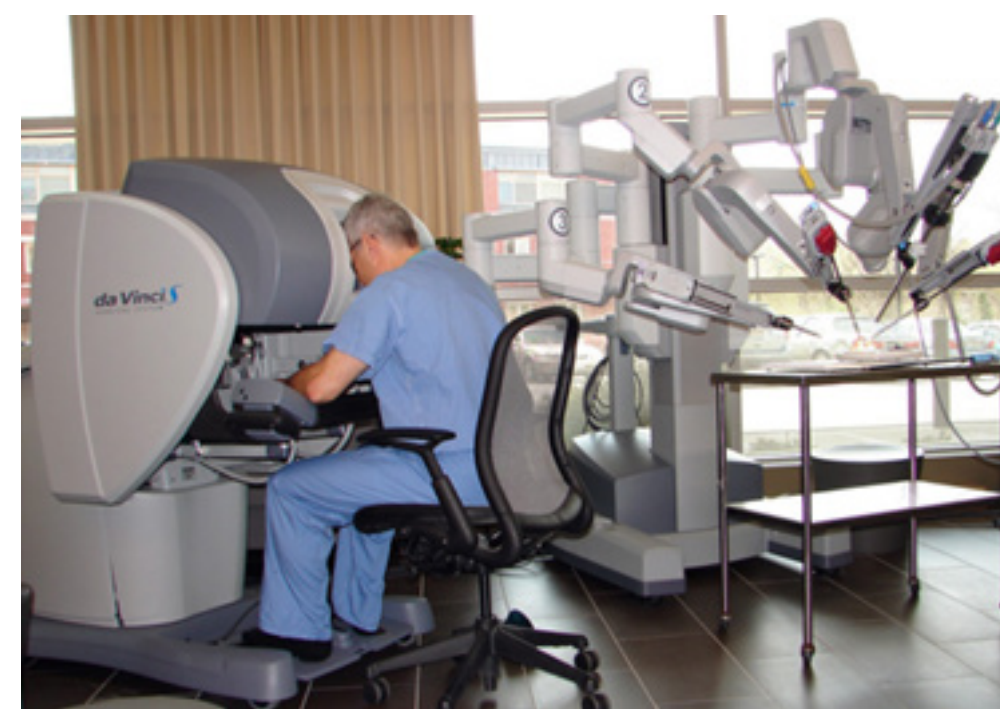

Imagen 1. Posicionamiento y Arquitectura de Da Vinci.

Fuente: Galeano, 2014. (Ob. Cit)

La cirugía Laparoscópica para muchos autores es considerada como la tecnología de transición para el nuevo paradigma robótico-quirúrgico que está siendo practicada, por su similitud en lógica instrumental. (Satava RM, 1999).

No obstante, una de las diferencias más importantes entre la laparoscopia y Da Vinci, es que éste último permite todos los de movimientos que asemeja a la muñeca. Porque mientras que los movimientos laparoscópicos son en dos dimensiones, los robóticos lo hacen en tres.

\section{Ventajas de la Cirugía Robótica frente a la Cirugía abierta y Laparoscopia}

- Permite al cirujano tener visión tridimensional a través del manejo de la cámara la cual aumenta 20 veces el tamaño real, lográndose así una imagen detallada de los órganos, cavidades y tejidos.

- Permite una mayor precisión en los movimientos instrumentado del cirujano, el cual puede articular los mismos con una adecuada ergonomía, precisión y filtro de temblor, porque el robot computa el movimiento.

- Ofrece una mayor libertad de movimiento articular de la muñeca del cirujano que en una cirugía laparoscópica tradicional. A través del robot, el cirujano realiza movimientos más precisos, acelera o reduce la velocidad de los movimientos de las manos, facilitándole las suturas y atar nudos.

- Disminuye el tiempo de estancia hospitalaria de los pacientes, porque el tiempo de convalecencia no es mayor de 7 días, ya que por ser mínimamente in-

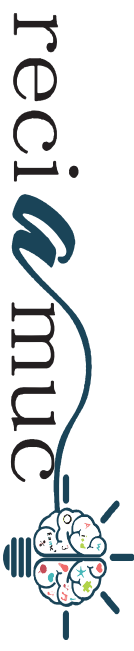




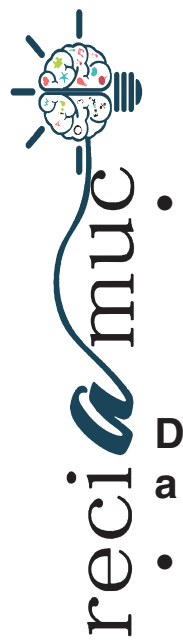

vasiva el paciente tiene menos pérdida sanguínea y con mejores resultados funcionales.

Cuando son robot de Telepresencia, permite operaciones a distancias remotas, evitando así, el desplazamiento tanto del paciente como del cirujano.

Desventajas de la Cirugía Robótica frente a la Cirugía abierta y Laparoscopia

- Es una tecnología demasiado costosa reduciéndose su accesibilidad a ciertas poblaciones de bajo recurso.

- Todavía son aparatosas y los robots ocupan demasiado espacio. Sin embargo, actualmente se vislumbra la minimización y mayor funcionabilidad.

- La sensación táctil del tejido por parte del cirujano es un reto para la robótica actual.

- En algunos robots, cuando se utiliza más de un brazo robótico, el movimiento puede ser limitado.

- Se reduce la participación de ayudantes quirúrgicos durante la cirugía.

- Por ahora, el tiempo de cirugía robótica es mayor que para la laparoscopia o la laparotomía tradicional, lo que aumenta el riesgo del paciente a la anestesia.

Actualmente, básicamente cualquier procedimiento laparoscópico puede ser realizado con cirugía robótica. Y definitivamente, esta es de mayor beneficio en los procedimientos que se realizan en campos reducidos o en cavidades de difícil acceso, como la pelvis o la cirugía reconstructiva.

En consecuencia, cada vez son más los médicos en cirugía general, en cirugía urológica, cirugía torácica, neurocirugía, ginecología, cardiovascular y cirugía vascular periférica, que se preparan para aprender y entrenarse con estos robots.

Curva de Aprendizaje en Cirugía General

\section{Robótica}

Actualmente, se incrementan los lugares en el mundo donde se realizan procedimientos quirúrgicos robóticos, y cada vez son menores los reportes de falla de los componentes del robot. Básicamente, cualquier cirugía que se pueda realizar por Laparoscopia, se puede realizar asistidos con robot. (Minor, Mosso, Domínguez, \& et al, 2002)

Sin embargo, una de las principales desventajas de la cirugía robótica es el costo que limita su difusión, sobre todo, en los países de menores recursos. También se considera una limitación a la cirugía robótica, la falta sensación táctil y el tamaño del equipo que resulta incómodo al ocupar tanto espacio. Aun así, los avances tecnológicos son muy rápidos y van de la mano de la demanda del mercado, por lo que es muy probable que en un futuro cercano estas dificultades sean resueltas.

El aprendizaje de esta nueva tecnología por parte de los cirujanos es considerado también una limitación. Los robots no son máquinas que pueden realizar toda la operación automáticamente, necesita de la experticia del cirujano para que el procedimiento se realice con éxito.

El concepto de curva de aprendizaje empezó a usarse en medicina en la década de los ochenta, cuando empezó a emerger la cirugía mínimamente invasiva. Una definición aceptable en la comunidad médica para la curva de aprendizaje consiste en "el tiempo y el número de procedimientos que un cirujano corriente necesita para ser capaz de realizar un procedimiento en forma independiente, con un resultado razonable" (Latiff, 2005)

Esto significa que el periodo durante el cual se adquiere la experticia suficiente para llevar a cabo un procedimiento con garantía de eficacia en resultados y seguridad, es un elemento importante para definir la curva de aprendizaje. Así como también lo es el número de casos con el cual el cirujano 
se sienta seguro y cómodo al realizar dicho procedimiento.

Determinar cuántos casos debe atender el cirujano realizando la operación robótica para poder considerarse como experto, o medianamente experto, es algo variable, que "muchas veces depende del nivel de autoexigencia del cirujano, de la experiencia previa en el procedimiento adaptado a la laparoscopia, del volumen de procedimientos realizados y de la habilidad personal" (Pereira Fraga, 2017).

Así pues, "la percepción de la curva de aprendizaje cambia con la experiencia previa, así cirujanos con experiencia en cirugía abierta las expectativas son más elevadas" (Herrel SD, 2005).

Realmente no existe un estándar aceptado para medir la curva de aprendizaje basado en esta variable, porque es finalmente el cirujano quién decide si tiene la experiencia suficiente para llevar a cabo una operación con ayuda robótica. Sin embargo, muchos autores estiman de 20 a 30 casos como mínimo para la curva de aprendizaje (Herrel SD, 2005).

Medir los resultados obtenidos en el paciente y el proceso clínico o eficiencia de la tarea, es una manera de evaluar la curva de aprendizaje. Así mismo, se ha considerado la incidencia de complicaciones y la sobrevida como indicadores para evaluación de la curva de aprendizaje (Latiff, 2005).

En definitiva, existen factores importantes que determinan la reducción de la curva de aprendizaje (Pereira Fraga, 2017):

- Actitud quirúrgica específica

- Destreza manual

- Adiestramiento estructurado sobre el sistema

Dichos factores son tomados en cuenta por diferentes estudios para realizar entre otras cosas la medición y evaluación de la Curva de Aprendizaje en cirugías realizadas con el Da Vinci.

Evaluación de la Curva de Aprendizaje en Cirugía General Robótica

A continuación se analizan diferentes estudios que han determinado la Curva de Aprendizaje en Cirugía General Robótica utilizado el método de conteo de casos y el tiempo como variable para determinarla:

1. En un estudio retrospectivo realizado en la Universidad de Illinois, entre agosto de 2000 y diciembre 2004, donde se evaluó la practica de cirugía robótica a 399 pacientes utilizando el Da Vinci Surgical System, se obtuvo como resultado una disminución de la curva de aprendizaje, donde se consideraba el tiempo operatorio y la morbilidad (Galvani \& Horgan, 2005).

Entre las cirugías robóticas realizadas en la Universidad de Illinois, en Chicago, se encuentran:

- 110 Bypassgástricos

- 30 Lap band (Banda gástrica ajustable)

- 59 Miotomías de Heller

- 12 Funduplicaturas de Nissen

- 6 Divertículos epifrénicos

- 18 Esofaguectomías totales

- 6 Reseccciones de leiomioma esofágico

- 1 Piloroplastia

- 2 Gastroyeyunostomías

- 2 Esfintero-plastias transduodenales

- 10 Adrenalectomías

- 145 Nefrectomías de donante vivo relacionado.

Cada caso tuvo su experiencia, sus resultados particulares en cuanto a ventaja o desventaja respecto a las intervenciones tradicionales y laparoscópica, y el aprendizaje obtenido por parte del cirujano y el equipo 
de operaciones. Así por ejemplo, inicialmente para las Funduplicaturas de Nissen y Lap band los tiempos operatorios fueron más largos que la intervención tradicional por laparoscopia. No obstante, después de disminuir la curva de aprendizaje, el tiempo operatorio y la morbilidad de ésas intervenciones quirúrgicas disminuyeron considerablemente.

C Asimismo se evidenció en los restantes procedimientos un aplanamiento de la curva de aprendizaje conforme al tiempo y el número de casos realizados.

2. Otro estudio realizado en el Quirófano "I" asignado al Programa de Cirugía Robótica del Hospital Universitario de Caracas (HUC), se comparó la curva de aprendizaje de sutura y anudado intracorpóreo sobre un modelo inanimado, en caja de entrenamiento mediante pinzas laparoscópicas convencionales versus laparoscopia asistida con el Sistema Da Vinci® S HD de Intuitive Surgical, en un periodo de 5 meses. La población objeto de estudio fueron 5 residentes con habilidades en cirugía laparoscópica básica, sin experiencia en sutura y anudado intracorpóreo. Concluyeron que las prácticas de tareas laparoscópicas complejas en sistemas robóticos mejoran y acortan la curva de aprendizaje en sujetos sin experiencia previa en laparoscopia avanzada (CAIBCO Centro de Análisis de Imágenes Biomédicas Computarizadas, 2018).

3. Finalmente, en la investigación denominada "What Is the Learning Curve in Robotic General Surgery?", se revisó una data en PubMed de 647 resúmenes de manuscritos publicados entre marzo de 1999 y julio de 2015, cuya literatura tratara sobre la curva de aprendizaje en cirugía general robótica, con la finalidad de informar a los usuarios de esta tecnología (Pernar, Robertson, Tavakkoli, Sheu, Brooks, \& Smink, 2017).

Después de los criterios de selección, sólo se incluyeron para el estudio 24 artículos, donde las muestras totales de cirugías del estudio variaron entre 32 y 200 casos, siendo las cirugías abordadas para estos, las siguientes:

- Cirugía Colorrectal: en 10 artículos (42\%)

- Cirugía de Intestino Anterior o Bariátrica: en 8 artículos (33\%),

- Cirugía de Órganos Sólidos:

- Biliar: en 2 artículos (8\%).

- Pancrática: en 4 artículos (17\%).

Los métodos utilizados para determinar la curva de aprendizaje fueron el número acumulado de casos (en 17 artículos, 71\%) y el tiempo se utilizó en un 100\% como variable para examinarla. El número de casos necesarios para aplanar la curva de aprendizaje no varió mucho de rango, inclusive entre los diferentes tipos de cirugía:

- Cirugía Colorrectal: 25 a 75 casos

- Cirugía de Intestino Anterior o Bariátrica: 10 a 95 casos

- Cirugía de Órganos Sólidos: 10 a 80 casos

Cabe destacar que 15 (63\%) de 24 artículos informan experiencias de un solo cirujano.

Finalmente, se puede afirmar que el método utilizado para evaluar la curva de aprendizaje en la mayoría de las cirugías robóticas es el de acumulación de casos a lo largo del tiempo. Sin embargo, en algunos estudios se han aplicado estrategias de enseñanzas para aplanar la curva siguiendo la experticia.

Así pues, algunos autores refieren que al realizar entre 8 y 12 procedimientos se adquiere la experiencia "equiparable a 100PR laparoscópicas para un cirujano sin experiencia previa en abordaje laparoscópico". (Ahlering , 2003)

Un estudio afirma que la sutura y anudado intracorpóreo logran ser un 65\% más rápidos al cabo de pocas prácticas de ense- 
ñanza (Yohannes P, 2002).

Otros estudios revelan que la curva de aprendizaje para el SETUP del sistema Da Vinci, pasa de 22,5 minutos en los primeros 10 casos a 8,5 minutos en los 10 siguientes, es decir se logra aplanar la curva (alcanzar la meseta ) en 30 casos (Majer E, 2007).

\section{Conclusión}

La evaluación de la Curva de Aprendizaje en Cirugía General robotizada que se utiliza en la actualidad, se basa en el método de acumulación de casos tomando al tiempo como variable para determinarla.

Para reducir los tiempos de aprendizaje el cirujano debe tener la experticia en cirugía laparoscópica y recibir entrenamiento sobre simuladores. Se estima que la Curva de Aprendizaje en el particular en el sistema Da Vinci, se aplana aproximadamente en 30 casos.

Definitivamente, el comportamiento de la Curva de Aprendizaje de la Cirugía Robótica tiende acortarse cada vez más, incluso para cirujanos sin experiencia en cirugía laparoscópica. Sin embargo, la demanda de aprendizaje es elevada y los métodos de enseñanza aún son limitados o insuficientes.

Además de entrenarse en las nuevas técnicas quirúrgicas, el cirujano debe contar con un amplio conocimiento sobre la patología a tratar, y un alto sentido humanitario que le permita priorizar la intervención más correcta, en el momento correcto, y con la herramienta más acertada para lograr que el paciente recupere su salud. En tal sentido los métodos de ensenanzas han de seguir profundizando, dada la vigencia y la proyección de estas técnicas quirurgicas, en el marco de ls enseñanza desde el udo adecuado y oportuno según los casos en estudio.

\section{Bibliografía}

Ahlering , T. E. (2003). Succesful transfer of open surgicals kills toa laparoscopic enviroment using robot interface: initial experience with laparoscopic radical prostatectomy. J Urol, 1738-1741.

Ballantyne G. (2002). Robotic surgery, telerobotic surgery, telepresence, and telementoring. Review of early clinical results. Surg Endosc, 16, 13891402.

CAIBCO Centro de Análisis de Imágenes Biomédicas Computarizadas. (2018). Sutura y anudado laparoscópico asistido por robot: estudio comparativo de la curva de aprendizaje. VITAE, Internet.

Cuschieri, A., Francis, N., Crosby, J., \& Hanna, G. (2001). What do master surgeons think of surgical competence and revalidation? Am J Surg, 182, 110-116.

Galeano, D. (10 de 2014). jeuazarru. Recuperado el 05 de 09 de 2020, de Robótica Médica: http:// jeuazarru.com/wp-content/uploads/2014/10/robotica_medicinal.pdf

Galvani, C., \& Horgan, S. (2005). Robots en cirugía general: presente y futuro. Cir Esp, 78(3), 138-147.

Herrel SD. (2005). Roboticassistedlararoscopicprostatectomy: Whatisthelearningcurve ? Urology, 66, 105-107.

Latiff, A. (2005). La "Curva de Aprendizaje": Qué es y cómo se mide. Rev Urología Colombiana, XIV(1), 15-17.

Majer E. (21 de 03 de 2007). AnnualEAU Congress. Berlin.

Minor, A., Mosso, J. L., Domínguez, A., \& et al. (2002). Robot para cirugía laparoscópica. Rev Mex Ing Biomed, 23, 27-32.

Morris B. (2005). Robotic surgery: applications, limitations, and impact on surgical education. Med Gen Med, 7(3), 72-75.

Pereira Fraga, J. G. (2017). Actualidad de la Cirugía Robótica. Rev. Cubana de Cirugía, 56(1), 50-61.

Pernar, L. I., Robertson, F., Tavakkoli, A., Sheu, E. G., Brooks, D. C., \& Smink, D. S. (14 de April de 2017). An apprasial of learning crve in robotic general surgery. Natonal Library of Medicinne.

Satava RM. (1999). Emerging technologies for surgery in the 21st century. Arch Surg, 134, 11971202.

Traxer, O., Gettman, M., \& Napper, C. (2001). The Impact of intense laparoscopic skills training on

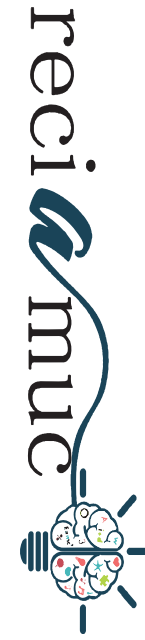


the operative performance of urology residents. J

Urol, 166, 1658-1661.

Yohannes P. (2002). Comparisonofroboticversus laparoscopicskills: istherea differencein thelearningcurve? Urlogy, 60, 39-45.

\section{(c) (1) $\circledast(0)$ BY NC SA}

RECONOCIMIENTO-NOCOMERCIAL-COMPARTIRIGUAL CC BY-NC-SA

ESTA LICENCIA PERMITE A OTROS ENTREMEZCLAR, AJUSTAR Y CONSTRUIR A PARTIR DE SU OBRA CON FINES NO COMERCIALES, SIEMPRE Y CUANDO LE RECONOZCAN LA AUTORÍA Y SUS NUEVAS CREACIONES ESTÉN BAJO UNA LICENCIA CON LOS MISMOS TÉRMINOS.

\section{CITAR ESTE ARTICULO:}

García Delgado, J., Morales Chaucalá, G., \& Vera Molina, H. (2020). Una evaluación de la curva de aprendizaje en cirugía general robótica. RECIAMUC, 4(2), 241-250. doi:0.26820/reciamuc/4.(2).abril.2020.241-250 